\title{
Norovirus genotypes in endemic acute gastroenteritis of infants and children in Finland between 1994 and 2007
}

\author{
L. PUUSTINEN ${ }^{1 *}$, V. BLAZEVIC ${ }^{1}$, L. HUHTI ${ }^{1}$, E. D. SZAKAL ${ }^{1}$, A. HALKOSALO ${ }^{1}$, \\ M. SALMINEN ${ }^{1}$ AND T. VESIKARI $I^{1,2}$ \\ ${ }^{1}$ Vaccine Research Center, University of Tampere Medical School, Tampere, Finland \\ ${ }^{2}$ Department of Pediatrics, Tampere University Hospital, Finland
}

(Accepted 9 March 2011; first published online 14 April 2011)

\section{SUMMARY}

Noroviruses are, after rotaviruses, the second most common causative agents of acute gastroenteritis in young children. We studied norovirus genotypes in faecal specimens collected from Finnish children followed-up prospectively in rotavirus vaccine trials. Almost 5000 faecal specimens collected from cases of acute gastroenteritis were examined using reverse transcriptase-PCR. A total of 1172 cases ( $25 \%$ of all acute gastroenteritis) were associated with noroviruses. Of these, $96 \%$ were genogroup GII. GII.4 was the most common genotype $(46 \%)$ throughout the study period but the proportion of this genotype varied in different norovirus epidemic seasons. Additional norovirus genotypes detected were: GII.7 (15\%), GII.3 (14\%), GII.1 (9\%), GII.b (7\%), GII.2 (3\%), and GI.3 (2\%). GII.4 dominated during the following years: 1998-1999 (75\%), 2002-2003 (88\%) and 2006-2007 (98\%) while recombinant genotype GII.b was dominant between 2003 and 2004 (83\%). In conclusion, genotypes GII.4 and GIIb have emerged as predominant norovirus genotypes in endemic gastroenteritis affecting young infants and children in Finland.

Key words: Gastroenteritis, Norwalk agent and related viruses, virology.

\section{INTRODUCTION}

Noroviruses (NoVs) are not only the leading causative agents of outbreaks of acute viral gastroenteritis worldwide in people of all ages, but also the second most common viral aetiological agents of severe childhood gastroenteritis after rotavirus $[1,2]$. Patel and co-workers [1] estimated that in children aged $<5$ years residing in resource-rich countries, NoVs cause $\sim 900000$ episodes of gastroenteritis

\footnotetext{
* Author for correspondence: L. Puustinen, M.Sc., University of Tampere Medical School, Vaccine Research Center, FM 3, Biokatu 10, FIN - 33520 Tampere, Finland. (Email: leena.puustinen@uta.fi)
}

necessitating a clinic visit, compared with resourcepoor countries in which NoVs may cause more than one million hospitalizations and up to 200000 deaths each year.

A high incidence of NoVs $(20 \%)$ in acute gastroenteritis (AGE) affecting young children was originally reported from Finland when stool specimens collected for rotavirus studies in connection with a rotavirus vaccine efficacy trial in 1993-1995 were also examined for human caliciviruses [3]. While many of the community-acquired $\mathrm{NoV}$ cases were mild, some were severe, and NoVs also accounted for about $10 \%$ of AGE cases seen in hospital [4]. In the present study, we examined the incidence and genotypes of NoVs in

The online version of this article is published within an Open Access environment subject to the conditions of the Creative Commons Attribution-NonCommercial-ShareAlike licence $<$ http://creativecommons.org/licenses/by-nc-sa/2.5/ $>$. The written permission of Cambridge University Press must be obtained for commercial re-use. 
Table 1. Study specimens analysed and the methods used for RNA extraction and NoV genotyping 1993-2007

\begin{tabular}{lccllll}
\hline \hline Study years & $\begin{array}{l}\text { No. of } \\
\text { samples }\end{array}$ & $\begin{array}{l}\text { NoV-positive } \\
\text { specimen, } N(\%)\end{array}$ & $\begin{array}{l}\text { Boom's } \\
\text { extraction }\end{array}$ & $\begin{array}{l}\text { QIAamp } \\
\text { Viral RNA } \\
\text { extraction }\end{array}$ & $\begin{array}{l}\text { Region A* } \\
\text { genotyping }\end{array}$ & $\begin{array}{l}\text { Region C* } \\
\text { genotyping }\end{array}$ \\
\hline 2007 & 106 & $57(54)$ & - & + & + & $+(53) \dagger$ \\
$2002-2004$ & 254 & $27(11)$ & - & + & + & - \\
$2001-2003$ & 569 & $90(16)$ & - & + & + & + \\
$2000-2002$ & 485 & $146(31)$ & + & - & + & + \\
$1998-2001$ & 1510 & $438(29)$ & - & + & + & - \\
$1997-1999$ & 326 & $101(31)$ & - & + & + & - \\
$1993-1995+$ & 1477 & $313(21)$ & + & $+(279)$ & $+(294)$ & - \\
Total & 4727 & $1172(25)$ & 1962 & 3044 & 1153 & 193 \\
\hline \hline
\end{tabular}

,- Not tested; +, tested.

* See Methods section and Figure 1.

$\dagger$ If not all specimens were tested the number in parentheses indicates the number of specimens tested.

\$ Results published in [4].

community-acquired AGE affecting young children during 1993-2007, a period when large numbers of infants and young children participated in several rotavirus vaccine trials in Finland.

Most NoVs infecting humans belong to genogroups GI and GII. GI genogroup is further subdivided into at least eight genotypes and GII genogroup into 17 genotypes [5]. Of the two main genogroups, GII is much more common worldwide $[6,7]$. Genotype GII.4 NoVs have predominated since the mid-1990s in the USA, Europe and Oceania, causing $70-80 \%$ of all NoV outbreaks in communities, nursing homes, schools, hospitals and cruise ships associated with contaminated food or water $[8,9]$. GII.4 has also commonly been detected in endemic infections of children worldwide [10-14]. Of the other genotypes, recombinant strain GIIb especially has also been frequently reported in paediatric NoV infections [11-13, 15].

In the past, NoV genotyping was performed solely on the RNA-dependent RNA polymerase (RdRp) region of open reading frame (ORF) 1 of the singlestranded, positive-sense NoV RNA genome [16]. Later studies showed better segregation of the different strains into their respective genotypes by phylogenetic analysis of nucleotide sequences within the capsid region of ORF2 [17]. However, genotyping based solely on the capsid sequence would miss the naturally occurring recombinant NoVs which cluster into two distinct groups of NoV strains when regions RdRp and capsid are subjected to phylogenetic analysis $[18,19]$. In this study, we genotyped NoVs according to polymerase region as well as to the capsid region.

\section{METHODS}

\section{Clinical specimens}

In total, 4727 faecal specimens were collected from AGE cases in children up to age 3 years participating in seven rotavirus vaccine efficacy trials in several different regions in Finland from 1993 to 2007 [3, 20-23]. The vaccine study protocols and consent forms had been approved by the appropriate ethics committees and patients or legal guardians volunteered for the study after having given informed consent. Stool specimens were collected from children receiving either placebo or rotavirus vaccine whenever an episode of AGE occurred. AGE was typically defined as $\geqslant 3$ looser than normal stools and/or vomiting within any day. Specimens collected within 14 days of the first sample from the same patient were considered to be duplicate samples and excluded from the analysis. The numbers of stool specimens from each year and the genotyping methods applied are described in detail in Table 1. Stool specimens were stored at $-20{ }^{\circ} \mathrm{C}$ until tested.

\section{RNA extraction}

For viral RNA extraction $10 \%(\mathrm{w} / \mathrm{v})$ stool suspensions were made in phosphate-buffered saline (PBS). Extractions were performed using the QIAamp ${ }^{\circledR}$ Viral RNA Mini kit (Qiagen, Germany) according to the manufacturer's instructions. In some cases (Table 1), RNA was extracted by binding to silica particles in the presence of guanidine thiocyanate as described earlier by Boom et al. [24]. Aliquots of 


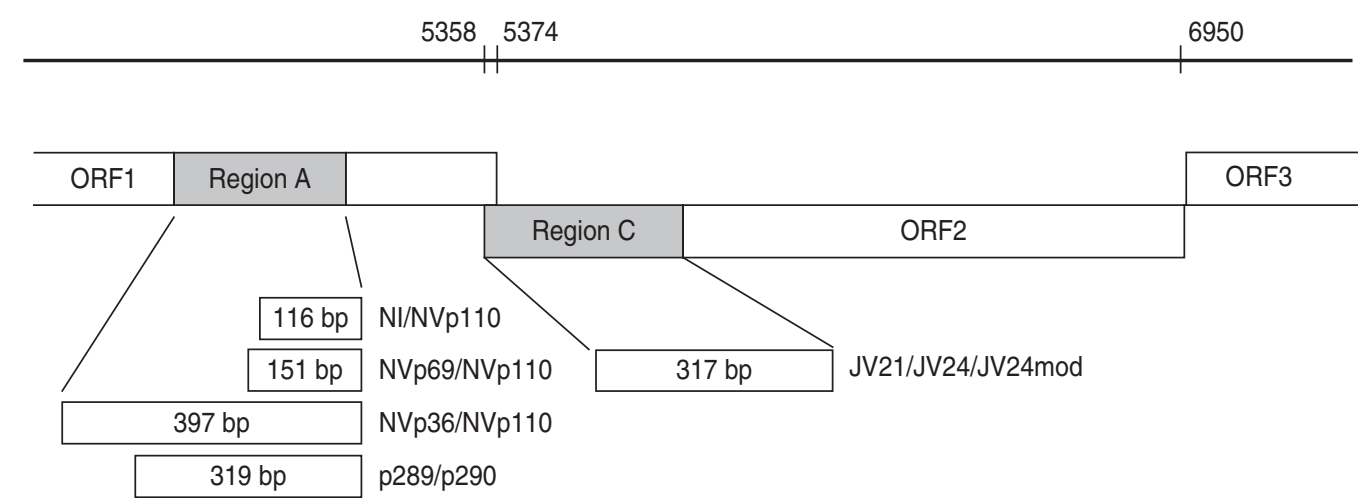

Fig. 1. Schematic presentation of norovirus genomic regions A and C (grey) [17], and RT-PCR primers (NI/NVp69/NVp36/ NVp110, p289H,I,IUB/p290H,I,J,K,IUB and JV21/JV24/JV24mod, described in the Methods section) used for reverse transcriptase (RT)-PCR and genotyping. Numbers on the bar refer to the nucleotide positions in Norwalk GI.1 virus genome (GenBank accession no. M87661). RT-PCR amplicon sizes in base pairs are noted in the boxes.

RNA extracts were stored at $-70{ }^{\circ} \mathrm{C}$ until reverse transcriptase (RT)-PCR testing.

\section{RNA polymerase (region A) RT-PCR}

For the specimens collected between 1994 and 1995 the detection of NoVs was performed using primer mixture sets including reverse primer $\mathrm{NVp110}$ and forward primers NI, NVp69 and Np36 (Fig. 1) [25]. These primers detect both genogroup I and II NoVs as well as sapovirus, and amplify $397 \mathrm{bp}, 151 \mathrm{bp}$ and 116 bp long PCR products from the RNA-dependent RNA polymerase ( $\mathrm{RdRp}$ ) region. RT-PCR reactions were performed as described previously [3].

For the specimens collected between 1997 and 2007, as well as for 279 samples from 1994 to 1995 (to confirm genotype findings done by the RT-PCR method described above), RNA polymerase region A detection was performed by the RT-PCR method reported by Jiang and co-workers [26], modified by Farkas and colleagues [27]. The primer mixture p289H, I/p290H,I,J,K was used with additional primers p289IUB (reverse: 5'-GATTACTCCARGTGGGAYTCMAC-3') and p290IUB (forward: 5'-TGACRATKTMATCATCMCCRTA-3') to improve detection of all genotypes. The RT reaction was performed at $42{ }^{\circ} \mathrm{C}$ for 60 min with $2 \cdot 5 \mu 1$ RNA added to the RT mixture (total volume $50 \mu \mathrm{l}$ ) containing $22.9 \mu 1$ sterile water, $1 \times$ GeneAmp PCR buffer (Applied Biosystems, USA), $1.5 \mathrm{~mm}$ GeneAmp $\mathrm{MgCl}_{2}$ (Applied Biosystems), $400 \mu \mathrm{M}$ dNTPs each, $16 \mathrm{ng} / \mu \mathrm{l}$ p289H,I,IUB reverse primer mixture, 10 U RNasin ${ }^{\circledR}$ (Promega, USA) and $70 \mathrm{U}$ M-MLV Reverse Transcriptase $\mathrm{RNase} \mathrm{H}^{-}$enzyme (Promega). Fifty microlitres of PCR reaction mixture consisting of $26.6 \mu \mathrm{l}$ sterile water, 2 U GoTaq DNA polymerase (Promega), $1 \times$ GoTaq Green buffer (Promega), $0.5 \mathrm{~mm} \mathrm{MgCl}_{2}$ (Promega), and a mixture of p290H,I,J,K,IUB forward primers $(24 \mathrm{ng} / \mu \mathrm{l})$ was added to the RT reaction. The 40-cycle PCR was run in a GeneAmp PCR system 9700 or Thermal Cycler 2720 (Applied Biosystems) with the following conditions: primary denaturation at $94{ }^{\circ} \mathrm{C}$ for $3 \mathrm{~min}$, denaturation at $94{ }^{\circ} \mathrm{C}$ for $30 \mathrm{~s}$, annealing at $42^{\circ} \mathrm{C}$ for $1 \min 30 \mathrm{~s}$, extension at $72{ }^{\circ} \mathrm{C}$ for $1 \mathrm{~min}$ and final extension at $72{ }^{\circ} \mathrm{C}$ for $10 \mathrm{~min}$. The PCR amplicons were analysed by agarose gel electrophoresis to confirm the correct size of the product. This RT-PCR method for the detection of RdRp of caliciviruses simultaneously detected both NoVs and sapoviruses and amplified a 319-bp amplicon for NoVs and a 331-bp amplicon for sapoviruses. Positive PCR products were stored at $-20{ }^{\circ} \mathrm{C}$ for sequencing.

\section{Capsid (region C) RT-PCR}

To confirm the genotyping by region A, RT-PCR typing targeted at region $\mathrm{C}$ (Fig. 1) from the beginning of the capsid region in ORF2 of NoVs was studied for 193 specimens collected between 2000-2002 and 2007 (Table 1). Five microlitres of RNA was first reverse-transcribed as described by Pang et al. [28], except that the reaction contained $1 \times$ first-strand buffer (Invitrogen, USA) and the final concentration of dNTPs was $375 \mu \mathrm{M}$ each. Synthesized cDNA was stored at $-20{ }^{\circ} \mathrm{C}$ unless used immediately for PCR reaction. A 317-bp fragment was amplified with primers JV21 (reverse), JV24 (forward) [29] and an additional forward primer JV24mod (5'-GTGAATGAAGATGGCGTCGA-3') 


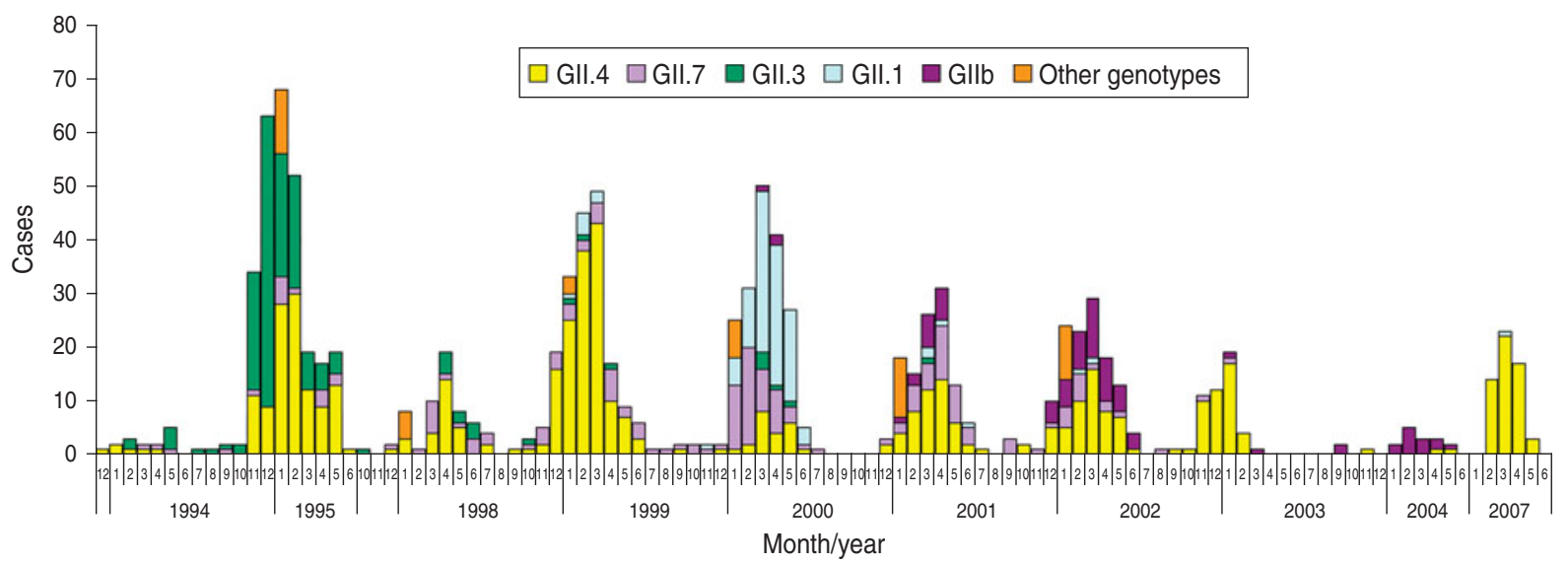

Fig. 2. Norovirus (NoV) seasonality and genotype distribution in Finnish children aged $<3$ years throughout the study period 1993-2007. From July 1995 to September 1997 and from June 2004 to January 2007 no specimens were collected.

for GII genotypes (Fig. 1). The PCR reaction was performed with $5 \mu \mathrm{l}$ cDNA added to PCR mixture (total volume $50 \mu \mathrm{l}$ ) consisting of $22.5 \mu 1$ sterile water, $2.5 \mathrm{U}$ GoTaq DNA polymerase (Promega), $1 \times$ GoTaq Green buffer (Promega), $1 \mathrm{~mm} \quad \mathrm{MgCl}_{2}$ (Promega), $200 \mu \mathrm{M}$ dNTP (Promega) and $4 \mathrm{ng} / \mu \mathrm{l}$ mixture of each of the above primers. The PCR run conditions were identical to those described above for RNA polymerase RT-PCR except that annealing temperature was $49^{\circ} \mathrm{C}$.

\section{Sequencing}

All the positive PCR products were excised from the electrophoresis gel, purified with QIAquick ${ }^{\circledR}$ Gel Extraction kit (Qiagen) according to the manufacturer's instructions and sequenced using the Big Dye ${ }^{\circledR}$ Terminator v. 1.1 Cycle Sequencing kit (Applied Biosystems). The primers used for sequencing were identical to those used in the RT-PCRs. Ethanol precipitation purified sequencing PCR products were analysed using an automated sequencer ABI PRISM ${ }^{\mathrm{TM}} 310$ Genetic Analyser (Applied Biosystems).

\section{Sequence analysis and genotyping}

Sequences obtained by the ABI PRISM 310 Genetic Analyser were aligned, verified and edited with the program Sequencher ${ }^{\mathrm{TM}} 4.8$ software (Gene Codes Corp., USA). Virus confirmation and genotyping was done using the Food-borne Viruses in Europe network (FBVE) NoV genotyping tool (http://www. rivm.nl/bnwww) and NCBI Blast ${ }^{\circledR}$ programs (http:// www.ncbi.nlm.nih.gov/BLAST/, nucleotide blast).
In addition, $\mathrm{RdRp}$ region $\mathrm{A}$ and capsid region $\mathrm{C}$ (Fig. 1) sequences of the recombinant strains were re-analysed with the new NoV genotyping tool of FBVE (http://www.rivm.nl; National Institute of Public Health and the Environment, The Netherlands) to confirm the genotyping.

\section{RESULTS}

A total of 4727 faecal specimens from AGE cases were studied and $1172(25 \%)$ cases were found to be associated with NoVs, as determined by genotyping according to region A sequences (Table 1). NoV genotypes during the period 1994-2007 by month are shown in Figure 2. The study period covered ten seasons with each season starting in July and ending in June. Of the 1149 genotyped NoV strains, 96\% were genogroup GII. GII.4 was the most common genotype throughout the study period $(46 \%)$, but it appeared in varying proportions in the NoV epidemic seasons (Fig. 2, Table 2). GII.4 was already present in community-acquired cases in 1993-1994 with a $40 \%$ share, but dominated in the seasons 1998-1999, 2002-2003 and 2006-2007 (Table 2, values in bold face).

Additional NoV genotypes detected are listed in Table 2. Other genotypes included: GI.6, GI.4, GII.9, GI.2, GIIU, GIId which accounted for $<1 \%$ each (Fig. 2, Table 2). During the seasons 1999-2000 and 2003-2004, which followed the GII.4 peak seasons, the shares of GII.4 were low $(13 \%$ and $17 \%$, respectively), and genotypes GII.1, GII.7 and GII.b dominated. In the early study years GII.4 was circulating with GII.3 (1994-1998) and with GII.7 (1994-2002), followed by GII.1 (1999-2000) and 
Table 2. Most commonly found NoV genotypes in sporadic cases of acute gastroenteritis in Finnish children 1993-2007. From July 1995 to September 1997 and from June 2004 to January 2007 there were no specimens available

\begin{tabular}{|c|c|c|c|c|c|c|c|c|c|c|c|}
\hline Season* ... & 93-94 & $94-95$ & $97-98$ & 98-99 & $99-00$ & $00-01$ & $01-02$ & $02-03$ & 03-04 & $06-07$ & \\
\hline Genotype & \multicolumn{10}{|c|}{ Percent of cases per season } & Total $(\%)$ \\
\hline GII.4 & 40 & 40 & 47 & 76 & 13 & 38 & 37 & 88 & 17 & 98 & 46 \\
\hline GII.7 & 20 & 5 & 22 & 15 & 29 & 27 & 12 & 6 & & & 15 \\
\hline GII.3 & 40 & 51 & 17 & 2 & 3 & 1 & & & & & 14 \\
\hline GII.1 & & & & 4 & 48 & 3 & 1 & & & 2 & 9 \\
\hline GII.b & & & & & 2 & 12 & 28 & 4 & 83 & & 7 \\
\hline GII.2 & & & 5 & 1 & & 8 & 12 & & & & 3 \\
\hline GI.3 & & & & 2 & 4 & 2 & 3 & 2 & & & 2 \\
\hline Other $\dagger$ & & 5 & 9 & 2 & 3 & 9 & 7 & & & & 4 \\
\hline$N \ddagger$ & 15 & 279 & 58 & 196 & 197 & 125 & 153 & 51 & 18 & 57 & 1149 \\
\hline
\end{tabular}

Bold values indicate peak years of GII.4 incidence.

* Season is a time period from July to June.

$\uparrow$ Other genotypes detected (GI.6, GI.4, GII.9, GI.2, GIIU, GIId and undetermined).

+ Total number of NoV-positive specimens (in italics).

GII.b in the later seasons (2000-2007). Overall, of the total ten study seasons GII.4 predominated in seven seasons, while other genotypes predominated only in one season each (Fig. 2, Table 2).

The second most common genotype throughout the 14 study years was GII.7, which appeared in multiple seasons but never dominated (Fig. 2, Table 2). Genotype GII.3 circulated largely in 1994-1995 (40-50\%) and 1997-1998 (17\%) but was not detected after 2001. Genotype GII.1 was detected only rarely in other seasons, but predominated in 2000. The first recombinant GII.b strains (GII.b-GII.2) appeared in March 2000. GII.b reached a high prevalence in 2001-2002 and was the dominating genotype in 2004. The most common GI strain GI.3 occurred during 1998-2003 with very low prevalence of 1-4\%. Only a few AGE cases with other GI strains (GI.6, GI.4, GI.2) were occasionally detected (data not shown). In 1994-2002 there were more than eight different genotypes circulating among the study children, but later, in 2002-2007, the number of different genotypes was at maximum three per season (Table 2).

To confirm whether genotyping by capsid region $\mathrm{C}$ would identify identical genotypes as determined by polymerase region $\mathrm{A}$, some of the specimens (years 2000-2002 and 2007, $n=193$ ) were genotyped for both of these regions (Fig. 1, Table 1). Identical genotyping results were obtained for both regions in all cases, except for the recombinant strains. Altogether 53 recombinant strains were found in 193 double
Table 3. Polymerase (region A) and capsid (region C) genotype combinations of recombinant strains detected in Finnish children in 2001-2002

\begin{tabular}{lll}
\hline \hline Region A & Region C & No. of cases (\%) \\
\hline GII.b & GII.3 & $41(77)$ \\
GII.b & GII.1 & $3(6)$ \\
GII.b & - & $2(4)$ \\
GII.7 & GII.6 & $6(11)$ \\
GII.7 & GII.14 & $1(2)$ \\
\hline \hline
\end{tabular}

-, Undetermined.

genotyped NoVs in 2001-2002. No recombinant strains were found in 2007, when GII.4 was detected almost exclusively (Tables 1 and 3). RNA polymerase genotype GII.b occurred most commonly with capsid genotype GII.3 (41 cases, $77 \%$ ) and rarely with GII.1 (three cases, 6\%). Polymerase type GII.7 occurred with capsid genotype GII.6 (six cases, $11 \%$ ) and GII.14 (one case, 2\%) (Table 3).

\section{DISCUSSION}

Rotavirus vaccination studies conducted in Finland with follow-up AGE specimens offered a unique opportunity to study extensively the prevalence of NoVs in community-acquired AGE in infants and young children. Due to genetic variation in NoVs, primer selection for RT-PCR assays has been challenging. 
It is possible that in early study seasons from 1993 to 1995 NoV-positive specimens were missed when we used polymerase region primers including NVp110 [25] which were shown to fail to detect some NoV strains [30]. However, the large number of samples allowed us to draw certain conclusions. The present study showed that in children aged $<3$ years, NoVs were responsible for about one quarter of AGE cases in the community.

Overall, GII.4 was the most prevalent genotype in young children during the study period 1994-2007, followed by GII.7, GII.3, GII.1 and GII.b. In European outbreaks of AGE in all ages 2001-2006 the most common genotypes were GII.4, GII.7, GII.2 and GII.b [8]. In European outbreaks GII.7 peaked in 2005-2006 [8] and in Finnish outbreaks in 2001 and 2002 [31]. However, in children we had already found GII.7 in earlier seasons (1997-1998 and 1999-2001). Consistent with the observations from outbreaks in Finland and other European countries [8, 11, 31] in winter 2001, GII.b was the third most common genotype, and its relative role increased until winter 2004 , when it was the predominant type $(83 \%)$. In contrast to our observation, in a Canadian study [32] no correlation was found between the NoV strains in the outbreaks and in sporadic childhood AGE, but the follow-up period was only a little over 1 year.

In our study, genogroup GI strains were uncommon and, therefore, appeared to be less common in sporadic cases in children than in outbreaks. In Finnish outbreaks in 1998-2002 GI strains were detected in $13 \%$, but we detected GI in $<6 \%$ [31]. On the other hand, both in the outbreaks and in our study, GI.3 was the most common GI genotype [31]. In general, GI strains occur throughout the year rather than seasonally, more often in water-mediated rather than person-to-person-mediated outbreaks, and only rarely in sporadic cases of NoV infections $[9,33-35]$.

The total proportion of GII. 4 NoV in young children was $46 \%$ for the years 1994-2007 and 52\% for 2001-2007. Genotype GII.4 has also been detected as the predominant NoV genotype in paediatric sporadic AGE cases reported elsewhere [11, 13, 14, 36]. The peaks of genotype GII.4 in winters 2002-2003 and 2006-2007 detected in Finland, occurred at the same time as peaks of GII.4 outbreaks elsewhere in Europe and the USA [8, 9]. In 1998-1999 GII.4 was also seen in Finnish outbreaks and this winter was also reported as a GII.4 outbreak peak year in Germany
[31, 37]. Furthermore, from 2006 to 2008 GII.4 was found in almost $90 \%$ of children hospitalized for NoV AGE (Räsänen et al., unpublished observations). An increase in NoV activity in outbreaks was seen in Finland and many other European countries in 2002 due to the emergence of a new GII.4 variant 2002 [8, 31, 37]. GII.4 variants 2006a and $2006 \mathrm{~b}$ were responsible for large NoV outbreaks in Finland in winter 2007 [38]. Analyses of genetic variants of GII.4 in Finnish children for the peak years are ongoing and will determine which subtypes were responsible for these peaks.

Naturally occurring recombination events are common in NoVs and the most common recombination site is the ORF1-ORF2 junction localized upstream of the capsid gene $[18,19]$. We found $77 \%$ of the recombinants in our study consisted of a combination of GII.b-GII.3, which was also the most common recombinant strain elsewhere and emerged recently as a causative agent for many outbreaks in Europe, Australia, and Asia [15, 19, 33]. Genotype GII.b was the most common genotype in Finnish outbreaks in 2001 but was not detected in Finland before January 2001 [34] nor elsewhere in Europe before August 2000 [35, 39]. In the present study six GII.b cases had already been detected in March-April 2000. This raised a new possibility regarding the origin and transmission of the recombinant GII.b strains, which were previously suspected to be in contaminated shellfish in August 2000 [39]. Children with sporadic gastroenteritis could serve as a reservoir for emerging epidemic NoV strains as some NoV strains appeared in children prior to emerging as epidemic outbreak strains $[13,36]$. To the best of our knowledge, we report for the first time the recombination of GII-7 polymerase with GII.14 capsid. However, the recombinant cases we described should be verified by sequencing over the ORF1-ORF2 junction region to exclude the possibility of mixed infections of different genotypes.

In conclusion, similar to findings worldwide, GII.4 was found to be the predominant NoV genotype in endemic gastroenteritis in infants and young children in Finland, at least since 1994. Recombinant genotype GII.b was detected with increasing incidence from March 2000 onwards. The shifts in NoV genotypes may be associated with an increase in the clinical significance of NoV AGE in young children overall, and warrant more in-depth studies on GII.4 variants. 


\section{ACKNOWLEDGEMENTS}

Dr Shang-Qin Zeng and the technicians at the laboratory of the Vaccine Research Center at the University of Tampere Medical School are gratefully acknowledged for their excellent technical assistance during the study.

\section{DECLARATION OF INTEREST}

None.

\section{REFERENCES}

1. Patel MM, et al. Systematic literature review of role of noroviruses in sporadic gastroenteritis. Emerging Infectious Disesses 2008; 14: 1224-1231.

2. Glass RI, Parashar UD, Estes MK. Norovirus gastroenteritis. New England Journal of Medicine 2009; 361 : 1776-1785.

3. Pang XL, Joensuu J, Vesikari T. Human calicivirusassociated sporadic gastroenteritis in Finnish children less than two years of age followed prospectively during a rotavirus vaccine trial. Pediatric Infectious Disease Journal 1999; 18: 420-426.

4. Pang XL, et al. Human caliciviruses in acute gastroenteritis of young children in the community. Journal of Infectious Diseases 2000; 181 (Suppl. 2): S288-94.

5. Zheng DP, et al. Norovirus classification and proposed strain nomenclature. Virology 2006; 346: 312-323.

6. Atmar RL, Estes MK. The epidemiologic and clinical importance of norovirus infection. Gastroenterological Clinics of North America 2006; 35: 275-90, viii.

7. Kroneman A, et al. Data quality of 5 years of central norovirus outbreak reporting in the European Network for food-borne viruses. Journal of Public Health (Oxford) 2008; 30: 82-90.

8. Kroneman A, et al. Analysis of integrated virological and epidemiological reports of norovirus outbreaks collected within the Foodborne Viruses in Europe network from 1 July 2001 to 30 June 2006. Journal of Clinical Microbiology 2008; 46: 2959-2965.

9. Zheng DP, et al. Molecular epidemiology of genogroup II-genotype 4 noroviruses in the United States between 1994 and 2006. Journal of Clinical Microbiology 2010; 48: 168-177.

10. Bucardo F, et al. Pediatric norovirus diarrhea in Nicaragua. Journal of Clinical Microbiology 2008; 46: 2573-2580.

11. Lindell AT, et al. Molecular epidemiology of norovirus infections in Stockholm, Sweden, during the years 2000 to 2003: association of the GGIIb genetic cluster with infection in children. Journal of Clinical Microbiology 2005; 43: 1086-1092.

12. Ramirez S, et al. Emerging GII.4 norovirus variants affect children with diarrhea in Palermo, Italy in 2006. Journal of Medical Virology 2009; 81 : 139-145.
13. Sdiri-Loulizi K, et al. Molecular epidemiology of norovirus gastroenteritis investigated using samples collected from children in Tunisia during a four-year period: detection of the norovirus variant GGII.4 Hunter as early as January 2003. Journal of Clinical Microbiology 2009; 47: 421-429.

14. Cheng WX, et al. Epidemiological study of human calicivirus infection in children with gastroenteritis in Lanzhou from 2001 to 2007. Archives of Virology 2010; 155: 553-555.

15. Bruggink LD, Marshall JA. Molecular and epidemiological features of GIIb norovirus outbreaks in Victoria, Australia, 2002-2005. Journal of Medical Virology 2009; 81 : 1652-1660.

16. Katayama K, et al. Phylogenetic analysis of the complete genome of 18 Norwalk-like viruses. Virology 2002; 299: 225-239.

17. Vinje J, Hamidjaja RA, Sobsey MD. Development and application of a capsid VP1 (region D) based reverse transcription PCR assay for genotyping of genogroup I and II noroviruses. Journal of Virological Methods 2004; 116: 109-117.

18. Bull RA, et al. Norovirus recombination in ORF1/ ORF2 overlap. Emerging Infectious Diseases 2005; 11: 1079-1085.

19. Bull RA, Tanaka MM, White PA. Norovirus recombination. Journal of General Virology 2007; 88: 33473359.

20. Vesikari T, et al. Safety, efficacy, and immunogenicity of 2 doses of bovine-human (UK) and rhesus-rhesushuman rotavirus reassortant tetravalent vaccines in Finnish children. Journal of Infectious Diseases 2006; 194: $370-376$.

21. Vesikari T, et al. Efficacy of RIX4414 live attenuated human rotavirus vaccine in Finnish infants. Pediatric Infectious Disease Journal 2004; 23: 937-943.

22. Vesikari T, et al. Effects of the potency and composition of the multivalent human-bovine (WC3) reassortant rotavirus vaccine on efficacy, safety and immunogenicity in healthy infants. Vaccine 2006; 24: 48214829.

23. Vesikari T, et al. Safety and efficacy of a pentavalent human-bovine (WC3) reassortant rotavirus vaccine. New England Journal of Medicine 2006; 354: 23-33.

24. Boom R, et al. Rapid and simple method for purification of nucleic acids. Journal of Clinical Microbiology 1990; 28: 495-503.

25. Le Guyader F, et al. Evaluation of a degenerate primer for the PCR detection of human caliciviruses. Archives of Virology 1996; 141: 2225-2235.

26. Jiang X, et al. Design and evaluation of a primer pair that detects both Norwalk- and Sapporo-like caliciviruses by RT-PCR. Journal of Virological Methods 1999; 83: 145-154.

27. Farkas T, et al. Genetic diversity among sapoviruses. Archives of Virology 2004;149: 1309-1323.

28. Pang XL, Preiksaitis JK, Lee B. Multiplex real time RT-PCR for the detection and quantitation of norovirus genogroups I and II in patients with acute 
gastroenteritis. Journal of Clinical Virology 2005; 33: 168-171.

29. Buesa J, et al. Molecular epidemiology of caliciviruses causing outbreaks and sporadic cases of acute gastroenteritis in Spain. Journal of Clinical Microbiology 2002; 40: 2854-2859.

30. Vinje $\mathbf{J}$, et al. International collaborative study to compare reverse transcriptase PCR assays for detection and genotyping of noroviruses. Journal of Clinical Microbiology 2003; 41: 1423-1433.

31. Maunula L, Von Bonsdorff CH. Norovirus genotypes causing gastroenteritis outbreaks in Finland 1998-2002. Journal of Clinical Virology 2005; 34: 186-194.

32. Lee BE, et al. Genetic relatedness of noroviruses identified in sporadic gastroenteritis in children and gastroenteritis outbreaks in northern Alberta. Journal of Medical Virology 2008; 80: 330-337.

33. Lysen M, et al. Genetic diversity among food-borne and waterborne norovirus strains causing outbreaks in Sweden. Journal of Clinical Microbiology 2009; 47: 2411-2418.
34. Maunula L, Miettinen IT, von Bonsdorff CH. Norovirus outbreaks from drinking water. Emerging Infectious Diseases 2005; 11: 1716-1721.

35. Bon F, et al. Molecular epidemiology of caliciviruses detected in sporadic and outbreak cases of gastroenteritis in France from December 1998 to February 2004. Journal of Clinical Microbiology 2005; 43 : 4659-4664.

36. Medici MC, et al. Molecular epidemiology of norovirus infections in sporadic cases of viral gastroenteritis among children in Northern Italy. Journal of Medical Virology 2006; 78: 1486-1492.

37. Lopman B, et al. Increase in viral gastroenteritis outbreaks in Europe and epidemic spread of new norovirus variant. Lancet 2004; 363: 682-688.

38. Kanerva M, et al. Prolonged norovirus outbreak in a Finnish tertiary care hospital caused by GII.4-2006b subvariants. Journal of Hospital Infection 2009; 71 : 206-213.

39. Ambert-Balay K, et al. Characterization of new recombinant noroviruses. Journal of Clinical Microbiology 2005; 43 : 5179-5186. 